\title{
Effect of routine seasonal malaria chemoprevention on malaria trends in children under 5 years in Dangassa, Mali
}

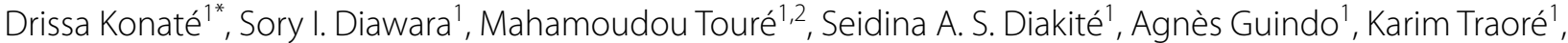 \\ Ayouba Diarra',2, Bourama Keita', Sibe Thiam', Moussa Keita', Ibrahim Sissoko', Nafomon Sogoba', \\ Sékou F. Traoré ${ }^{1,2}$, Donald J. Krogtad ${ }^{3}$, Seydou Doumbia ${ }^{1,2}$ and Mahamadou Diakité ${ }^{1,2}$
}

\begin{abstract}
Background: Seasonal malaria chemoprevention (SMC) is a new strategy to prevent malaria in children under 5 years old. It has been recommended by the World Health Organization since 2012 in malaria-endemic areas with seasonal transmission. This study aimed to assess the changes in malaria indicators through two consecutive years of SMC routine implementation in children under 5 years old in Dangassa, where malaria is endemic with a long and high transmission season.

Methods: From 2012 to 2016, a cohort study was conducted in Dangassa village. The study team based in the village followed all malaria clinical cases in children under 5 years old at the community health centre. During the study, SMC was routinely implemented in collaboration with the National Malaria Control Programme. The Cox regression model was used in order to compare malaria risk during the study.

Results: The Cox regression model showed a significant reduction in malaria clinical incidence, both in 2015 $(\mathrm{HR}=0.27(0.18-0.40), 95 \% \mathrm{Cl})$ and in $2016(\mathrm{HR}=0.23(0.15-0.35), 95 \% \mathrm{Cl})$ of SMC implementation compared to October 2013. Gametocyte and fever prevalence was lower between September and October during SMC implementation (2015 and 2016) compared to the same period before SMC implementation (2013-2014). A slight increase of malaria incidence was observed in December at the end of SMC implementation.
\end{abstract}

Conclusion: SMC has significantly reduced both malaria incidence and gametocyte prevalence and improved haemoglobin levels in children under 5 years old after 2 years of routine implementation.

Keywords: Children, Malaria indicators, SMC, NMCP, Mali

\section{Background}

Malaria represents an important public health problem in Africa with the highest morbidity and mortality rates in children under 5 years old [1]. Despite the wide deployment of malaria control interventions, the

\footnotetext{
*Correspondence: dkonate@icermalli.org

${ }^{1}$ West African International Center for Excellence in Malaria Research

(ICEMR-WA), University of Sciences, Techniques and Technologies of Bamako, Bamako, Mali

Full list of author information is available at the end of the article
}

sub-Saharan Africa region continues to carry a disproportionately high share of the malaria burden, which constitutes a barrier for economic and social development [2, 3]. In Mali, the national prevalence of malaria is $36 \%$ in children under 5 years old [4] despite the implementation of malaria control interventions such as intermittent preventive treatment with sulfadoxine-pyrimethamine in pregnancy (IPTp) and long-lasting insecticide-treated bed nets (LLINs) [5].

The World Health Organization (WHO) recently recommended seasonal malaria chemoprevention (SMC) in

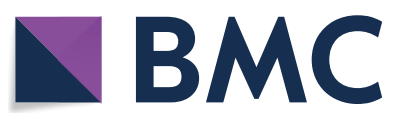

(c) The Author(s) 2020. This article is licensed under a Creative Commons Attribution 4.0 International License, which permits use, sharing, adaptation, distribution and reproduction in any medium or format, as long as you give appropriate credit to the original author(s) and the source, provide a link to the Creative Commons licence, and indicate if changes were made. The images or other third party material in this article are included in the article's Creative Commons licence, unless indicated otherwise in a credit line to the material. If material is not included in the article's Creative Commons licence and your intended use is not permitted by statutory regulation or exceeds the permitted use, you will need to obtain permission directly from the copyright holder. To view a copy of this licence, visit http://creativeco mmons.org/licenses/by/4.0/. The Creative Commons Public Domain Dedication waiver (http://creativecommons.org/publicdomain/ zero/1.0/) applies to the data made available in this article, unless otherwise stated in a credit line to the data. 
children under 5 years old, in additional to key interventions for malaria control in sub-Saharan Africa region. It consists to monthly administration of curative dose of sulfadoxine-pyrimethamine (SP) + amodiaquine (AQ) during 4 months in areas with highly transmission season [6]. In Mali, SMC is conducted from July to October according the National Malaria Control Programme (NMCP) recommendation. Beyond its actual efficacy on uncomplicated and severe malaria cases in the target population, this strategy may also impact the whole malaria epidemiology in areas where it is implemented $[7,8]$. However, the efficacy of this strategy has been reported in areas where malaria transmission lasts longer only during the SMC period (from July to September) [9], but not after stopped the drug administration in this area where malaria transmission is intense and lasts for 6 consecutive months annually [10].

There is little information on the effect of SMC strategy on malaria epidemiology in areas with long seasonal transmission. Information on malaria trends in these areas is important for the NMCP planning regardless of the variability of malaria endemicity.

In this study, the effect of SMC on malaria was assessed in children during an SMC intervention and 2 months after drug administration was stopped in the village of Dangassa, an area with intense and long seasonal malaria transmission.

\section{Methods}

\section{Study site}

This study is part of the International Centre for Excellence in Malaria Research (ICEMR) project in West Africa. The main goal of this project was to assess the effect of malaria control interventions on malaria epidemiology. The village of Dangassa is one of the ICEMR project sites in Mali since 2012. Dangassa is located approximately at $80 \mathrm{~km}$ southwest of Bamako. The main village is located $4 \mathrm{~km}$ from the Niger River (Fig. 1, map of Dangassa village). Malaria transmission is seasonal and occurs during 5 to 6 months per year. The

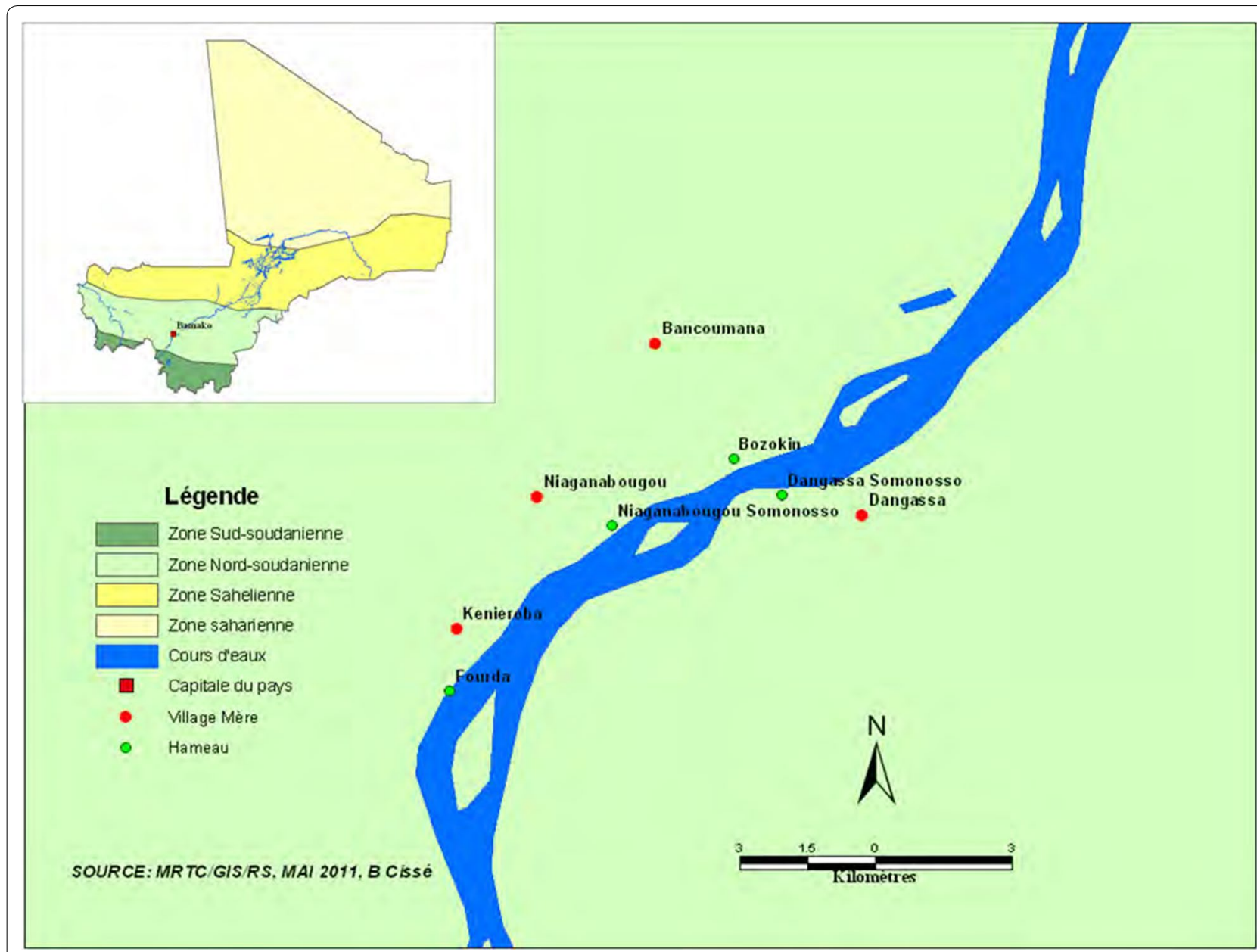

Fig. 1 Map of Dangassa village 
total population of Dangassa is about 6800 inhabitants; Malinke represents the main ethnic group [census data from geographic information system/malaria research \& training centre (GIS/MRTC) in 2015]. The study team was based at the community health centre in Dangassa for malaria cases detection.

During the rainy season, mosquito breeding in Dangassa occurs primarily in microhabitats such as the footprints of cattle and natural and man-made pools. During the dry season, as the river is receding, natural pools from the river as well as gold mining pools are formed which serve as breeding sites for mosquitoes, thus extending the transmission season [11].

\section{Study design and population}

Since the start of the ICEMR project in 2012, a dynamic study cohort has been selected from Dangassa population in order to assess the effect of control interventions on malaria epidemiology. This cohort included at least 1400 volunteers randomly selected from the census database with the assumption of an average number of 6 persons per household according demographic and health survey of Mali; about 240 households were selected for the cohort of 1400 children and adults. Children under 5 years of age represented $32.6 \%$ of the cohort population. An identification card was designed for all study participants with a unique ID number and a photo ID. Parents of the children were asked to present this identification card during each survey and health centre visit in order to facilitate their identification during the followup by the study team. In 2012, 450 children were enrolled in the main cohort and followed yearly for malaria case detection. A new enrolment of children at 3 months of age was done each year before the transmission season for census updated.

Follow-up of participants began in 2013 by the study team based at the Dangassa health centre for passive case detection of malaria. Each participant received at the health centre with malaria symptoms (e.g., temperature $>37.5{ }^{\circ} \mathrm{C}$ ), a blood sample was taken in order to determine parasitaemia and haemoglobin level. In addition, demographic information (age, gender, ethnic group), and bed net ownership and use (during the consultation at health centre) were collected also. The malaria episode was defined as fever and the presence of Plasmodium falciparum in the blood smear using microscopy. Anaemia was defined as a haemoglobin level $<11 \mathrm{~g} / \mathrm{dl}$ using the Hemocue ${ }^{\circledR} 301$ device.

\section{Implementation of routine seasonal malaria chemoprevention}

The aim of this study was to determine SMC effect on malaria indicators in children under 5 years old living in
Dangassa. During the ICEMR project, a routine SMC was implemented in children under 5 years old during 2015 and 2016 transmission seasons in collaboration with the NMCP. A monthly curative dose of SP $+\mathrm{AQ}$ was given to each child in 2015 and in 2016 (SMC) during malaria transmission season (August to October). In this study, 2013 and 2014 were considered as control (2 years prior to SMC implementation = no SMC). In 2015 and 2016, all children under 5 years in the cohort study received three doses of $\mathrm{SP}+\mathrm{AQ}$ from August to October at the community health centre. The sample size of this study is based on feasibility considerations for both epidemiological and entomological studies assuming a confidence level of $95 \%$. The data from September to December during 2013-2016 malaria transmission seasons were collected on case detection for this study. Before SMC implementation in Dangassa in 2015, the NMCP organized a mosquito net distribution campaign at the start of 2014.

\section{Data collection and analysis}

Data were collected on case report forms (CRF), entered on Microsoft Excel 2017 and analysed using Stata 14. During this study, data were collected on malaria incidence (person/month), fever prevalence, gametocyte prevalence, and haemoglobin level. Cox regression model was used to compare the risk of malaria first episode from September to December during the four malaria transmission seasons with a schedule at 5\%. Data from 2014, 2015 and 2016 were compared to those of 2013 (baseline).

\section{Results}

Fever prevalence decreased in September (49.3\%, $\mathrm{p}=0.004)$ and October $(55.1 \% ; \mathrm{p}=0.005)$ during SMC implementation (2015-2016) compared to September and October before SMC. However, it remains similar in November $(p=0.17)$ and December $(p=0.19), 2$ months after stopping drug administration (Fig. 2).

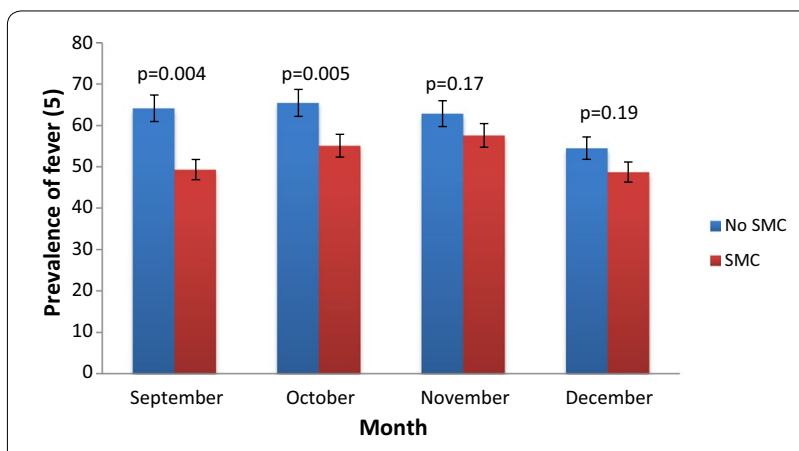

Fig. 2 Monthly prevalence of all fever in children under 5 years old in Dangassa from September to December 2013-2016 
A decrease of gametocyte prevalence was observed in September $(2.4 \% ; \mathrm{p}=0.001)$ and October $(8.8 \%$; $\mathrm{p}=0.001$ ) during SMC implementation compared to September and October 2013-2014 (no SMC) (15.2\% and $16.6 \%$, respectively). In November and December, the gametocyte prevalence remains similar SMC vs no SMC groups (Fig. 3).

A significant increase of haemoglobin level was observed in the second year (2016) of SMC implementation in September $(\mathrm{p}=0.01)$, October $(\mathrm{p}=0.001)$ and November $(\mathrm{p}=0.001)$ (Table 1$)$. But it's was observed only in October and November 2015.

Malaria incidence decreased from September (5.2 vs $10.2 \% ; \mathrm{p}=0.001)$, October (5.2 vs 9.3\%; $\mathrm{p}=0.001)$ and November (5.7 vs 9.2\%; $\mathrm{p}=0.001$ ) compared to the same period before SMC implementation. However, malaria clinical incidence remains similar in December (8 vs 9\%; $\mathrm{p}=0.2$ ), 2 months after stopping drug administration (Fig. 4).

Cox regression model showed after adjusted for insecticide-treated nets (ITN) use, a significant reduction of malaria clinical risk in September $(65 \%, \mathrm{p}=0.001)$, October $(73 \%, \mathrm{p}=0.001)$ and November $(62 \%, \mathrm{p}=0.001)$ in 2015. During the second year of SMC implementation, $75 \%(\mathrm{p}=0.001)$ of reduction in malaria clinical risk were observed in September, 77\% $(\mathrm{p}=0.001)$ in October and $63 \%(\mathrm{p}=0.001)$ in November. However, the risk was similar in December, 2 months after stopping drug administration $(\mathrm{p}=0.4, \mathrm{p}=0.5$ and $\mathrm{p}=0.1)($ Table 2$)$.

\section{Discussion}

The WHO recommends SMC in sub-Saharan Africa Region where malaria season transmission is short and high. In Mali, SMC has been implemented in some health districts since 2012 but a pilot study was conducted in Dangassa village in 2015. The goal of this study was to assess the effects of SMC on malaria trends in children living in Dangassa area were malaria transmission season

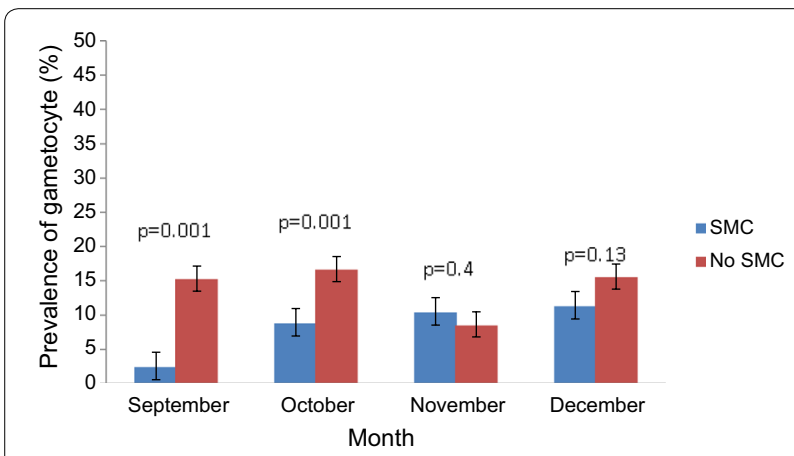

Fig. 3 Monthly prevalence of gametocyte in children under 5 years in Dangassa from September to December 2013-2016
Table 1 Hemoglobin level in children less than 5 years in Dangassa from September to December 2013-2016

\begin{tabular}{llrll}
\hline Years & \multicolumn{4}{l}{ Hemoglobin level in g/dl } \\
\cline { 2 - 5 } & September & \multicolumn{1}{l}{ October } & November & December \\
\hline 2013 & $10.8 \pm 0.1$ & $9.8 \pm 0.2$ & $10.4 \pm 0.2$ & $10.5 \pm 0.2$ \\
2014 & $10.2 \pm 0.1$ & $10.4 \pm 0.1$ & $10.4 \pm 0.1$ & $10.7 \pm 0.2$ \\
2015 & $10.6 \pm 0.1$ & $10.0 \pm 0.1$ & $10.8 \pm 0.1$ & $10.6 \pm 0.1$ \\
2016 & $10.9 \pm 0.1$ & $10.7 \pm 0.1$ & $10.9 \pm 0.1$ & $10.7 \pm 0.1$ \\
\hline
\end{tabular}

length is extended by the presence of the Niger River. The data were collected in children enrolled in a main study cohort during malaria case detection from September to December 2013-2016.

Data showed a significant decrease of fever prevalence in September and October during SMC period but remains similar in November and December between SMC vs no SMC (Fig. 2). After two consecutive years of SMC implementation (2015 and 2016), a decrease in gametocyte carriage was observed in September and October in SMC group (Fig. 3). An improvement of haemoglobin level (Table 1) was observed over the intervention period (September to November). The above observations could be attributed to SMC as it has been shown in many other studies about the protective effect of SMC on malaria in children in Africa [12-14]. The regular monthly administration of $\mathrm{SP}+\mathrm{AQ}$ allows maintenance of drug concentration in blood and prevents parasite growth and avoids red blood cell destruction, which in turn prevents haemoglobin level decrease by malaria parasites $[14,15]$.

A decrease in malaria incidence was also observed over the drug administration period in both 2015 and 2016 . However, a moderate increase in malaria incidence was observed in December, 2 months after the SMC campaign in October in both years 2015 and 2016 (Fig. 4). IPT during the malaria transmission season improved

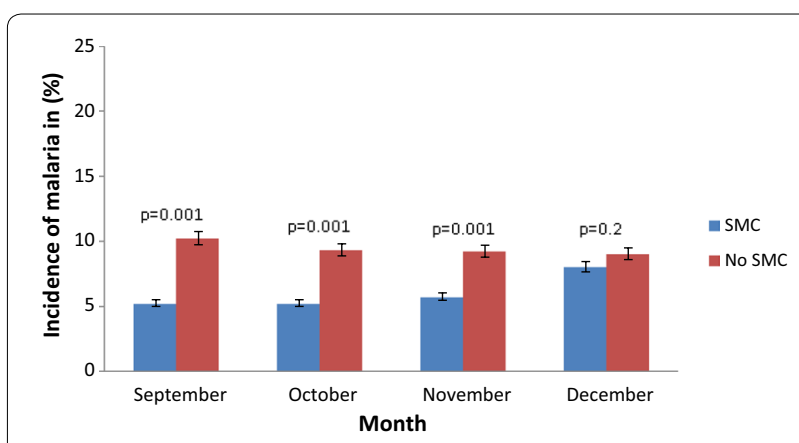

Fig. 4 Monthly incidence rate of malaria in children under 5 years from September to December in Dangassa 2013-2016 
Table 2 Risk of malaria first episode in children under 5 years from September to December 2013-2016 in Dangassa

\begin{tabular}{|c|c|c|c|c|c|c|c|c|c|c|c|c|}
\hline \multirow[t]{2}{*}{ Variables } & \multicolumn{3}{|c|}{ September } & \multicolumn{3}{|c|}{ October } & \multicolumn{3}{|c|}{ November } & \multicolumn{3}{|c|}{ December } \\
\hline & HR & IC 95\% & $p$ & HR & IC 95\% & $p$ & $H R$ & IC 95\% & $p$ & HR & IC 95\% & $p$ \\
\hline \multicolumn{13}{|l|}{ Years } \\
\hline 2013 & 1 & (Base) & & 1 & (Base) & & 1 & (Base) & & 1 & (Base) & \\
\hline 2014 & 0.79 & {$[0.51-1.21]$} & 0.2 & 0.60 & [0.39-0.92] & 0.02 & 0.73 & {$[0.50-10.5]$} & 0.09 & 0.83 & [0.49-1.40] & 0.4 \\
\hline 2015 & 0.35 & {$[0.22-0.53]$} & 0.001 & 0.27 & {$[0.18-0.40]$} & 0.001 & 0.38 & {$[0.25-0.57]$} & 0.001 & 0.86 & [0.49-1.49] & 0.5 \\
\hline 2016 & 0.25 & {$[0.16-0.37]$} & 0.001 & 0.23 & {$[0.15-0.35]$} & 0.001 & 0.37 & {$[0.25-0.55]$} & 0.001 & 0.70 & {$[0.43-1.14]$} & 0.1 \\
\hline \multicolumn{13}{|l|}{ ITN USE } \\
\hline No & 1 & (Base) & & 1 & (Base) & & 1 & (Base) & & 1 & (Base) & \\
\hline Yes & 1.18 & {$[0.86-1.62]$} & 0.2 & 1.22 & [0.93-1.6] & 0.1 & 1.07 & [0.83-1.37] & 0.5 & 1.45 & [1.05-1.99] & 0.02 \\
\hline
\end{tabular}

malaria epidemiological trends in children [16]. A substantial reduction of malaria indicators in children was already reported in Mali $[8,12]$ and elsewhere in Africa $[14,17,18]$, which was attributed to SMC implementation in addition to other control interventions, such as IPT and LLINs. The beneficial effect of SMC on malaria clinical incidence has been reported by many other studies $[15,19,20]$. The increase of malaria clinical incidence in December, 2 months after the SMC campaign, could be explained by local epidemiological conditions (proximity to the Niger River and rainfall with abundance of larval breeding) that has an effect on malaria transmission after the rains stop, and also the adherence of population to SMC. Stopping SMC provision while transmission continues could expose children at higher risk because the administrations of drugs to prevent malaria can slowdown the building of their immunity against malaria parasites [10].

The above statements were supported by the results of Cox regression model, which showed a significant reduction of malaria incidence from September to November in both 2015 and 2016 compared to 2013 after adjustment to bed net use. The risk of malaria was similar in 2014, the second year of study cohort without SMC. However, the risk of malaria clinical incidence remained similar in December, 2 months after drug administration was stopped (Table 2). These results suggest the protective effect of SP + AQ on malaria parasite growing [21] but showed that SMC could not have a beneficial effect on malaria trends after stopping drug administration.

The limitation of this study is the not availability of information on other ongoing interventions that could have an effect on malaria trends (e.g., use of ITNs and proper management of malaria cases). Therefore, these results cannot be attributed solely to SMC because it is an additional intervention to others, such as IPTp with SP, the use of ITN (distribution campaign at the start of 2014) and proper management of malaria cases in this area. It would be advisable to develop an appropriate approach for routine implementation of SMC in areas where seasonal transmission of malaria is long and prolonged by the existence of other conditions favouring transmission, as is the case with the Niger River and Dangassa. This is necessary for countries such as Mali where the Niger River and its tributaries cover long distances, in order to prepare targeted interventions necessary for malaria elimination. In addition, a better understanding of the adverse effects associated with amodiaquine metabolism, drug concentration and drug resistance on the systematic implementation of SMC in Mali is essential for its success in malaria-endemic countries.

\section{Conclusion}

This study has shown that SMC implementation in conjunction with other control intervention have significantly reduced malaria indicators in children during SP/ AQ drug administration in Dangassa village.

\section{Abbreviations}

AQ: Amodiaquine; CRF: Case report form; ICEMR: International Center for Excellence in Malaria Research; ITN: Insecticide-treated nets; LLIN: Long-lasting insecticide-treated net; NMCP: National Malaria Control Programme; SMC: Seasonal malaria chemoprevention; SP: Sulfadoxine-pyrimethamine; WHO: World Health Organization.

\section{Acknowledgements}

We thank all volunteers and the parents and guardians of children who participated in this study; DSK is supporting by the Fogarty International Center Grant number to SD (Grant\# D43TW008652).

\section{Authors' contributions}

DSK, SID, MT, SAS, AG, AD, BK, ST, MK, IS, NS, SFT, DJK, SD, and MD designed the study; DSK, SID, MT, SAS, AG, AD, BK, ST, MK, IS, NS, and MD coordinated and carried out field activities. DSK, SID, MT, and MD performed data analyses; DSK prepared initial draft of the manuscript; MT, SD, NS, and MD performed critical review and revision of the manuscript. All authors read and approved the final manuscript.

\section{Funding}

The ICEMR project is sponsored by the National Institute of Allergy and Infectious Diseases (NIAID). This project is funded by Division of Microbiology and Infectious Diseases, NIAID, NIH, Grants U19AI 089696-01 WEST AFRICA ICEMR 
AWARD. Protocol DMID Number: 11-0028. The funders had no role in study design, data collection and analysis, decision to publish, or preparation of the manuscript.

\section{Availability of data and materials}

For any information for the data presented here, please contact the corresponding author.

\section{Ethic approval and consent to participate}

Ethical clearance for this study was provided by the Ethics Committee of the Faculty of Medicine, Pharmacy, and Odontostomatology, University of Bamako ( $\left.N^{\circ} 2011 / 77 / F M P O S\right)$. Written informed consent was obtained from all participants aged $\geq 18$ years, or the parents or guardians of individuals aged $<18$ years. In addition, written assent was obtained from children aged 12 to 17 years prior the enrollment in the study.

\section{Consent for publication}

All authors read and approved the final revised manuscript.

\section{Competing interests}

The authors declare that they have no competing interests.

\section{Author details}

${ }^{1}$ West African International Center for Excellence in Malaria Research (ICEMR-WA), University of Sciences, Techniques and Technologies of Bamako, Bamako, Mali. ${ }^{2}$ University Clinical Research Center, University of Sciences, Techniques and Technologies of Bamako, Bamako, Mali. ${ }^{3}$ Tulane University School of Public Health and Tropical Medicine, New Orleans, LA, USA.

Received: 31 October 2019 Accepted: 23 March 2020

Published online: 06 April 2020

\section{References}

1. WHO. World malaria report 2018. Geneva: World Health Organization; 2018. https://apps.who.int/iris/bitstream/handle/10665/275867/97892 41565653-eng.pdf. Accessed 24 Nov 2019.

2. Audibert M. Lutte contre le paludisme: approche économique des obstacles à son contrôle. Sciences Sociales et Santé. 2004;22:25-33.

3. Bowden S, Michailidou D, Pereira A. Chasing mosquitoes: an exploration of the relationship between poverty, economic growth and the elimination of malaria in southern europe in the twentieth century. J Int Dev. 2008;20:1080-106.

4. NMCP, Institut National de la Statistique, ICF International. Enquête sur les Indicateurs du Paludisme au Mali (EIPM). Bamako; 2015. https://dhspr ogram.com/pubs/pdf/MIS24/MIS24.pdf. Accessed 24 Sept 2019.

5. NMCP. Plan stratégique de lutte contre le paludisme 2013-2017. Bamako; 2013. http://mail.cnom.sante.gov.ml/docs/PStrag\%202013-17PNLP.pdf. Accessed 24 Sept 2019.

6. WHO. World Malaria Report 2012. Geneva: World Health Organization; 2012. https://www.who.int/malaria/publications/world_malaria_repor t_2012/report/en/. Accessed 24 Sept 2019.

7. Dicko A, Diallo Al, Tembine I, Dicko Y, Dara N, Sidibe Y, et al. Intermittent preventive treatment of malaria provides substantial protection against malaria in children already protected by an insecticide-treated bednet in Mali: a randomised, double-blind, placebo-controlled trial. PLoS Med. 2017;8:e1000407.

8. Thera MA, Kone AK, Tangara B, Diarra E, Niare S, Dembele A, et al. Schoolaged children based seasonal malaria chemoprevention using artesunate-amodiaquine in Mali. Parasite Epidemiol Control. 2018;3:96-105.

9. Barry A, Issiaka D, Traore T, Mahamar A, Diarra B, Sagara I, et al. Optimal mode for delivery of seasonal malaria chemoprevention in Ouelessebougou, Mali: a cluster randomized trial. PLoS ONE. 2018;13:e0193296.

10. Greenwood BM, David PH, Otoo-Forbes LN, Allen SJ, Alonso PL, Armstrong Schellenberg JR, et al. Mortality and morbidity from malaria after stopping malaria chemoprophylaxis. Trans R Soc Trop Med Hyg. 1995;89:629-33.

11. Sogoba N, Doumbia S, Vounatsou P, Baber I, Keita M, Maiga M, et al. Monitoring of larval habitats and mosquito densities in the Sudan savanna of Mali: implications for malaria vector control. Am J Trop Med Hyg. 2007;77:82-8.

12. Diawara F, Steinhardt LC, Mahamar A, Traore T, Kona DT, Diawara H, et al. Measuring the impact of seasonal malaria chemoprevention as part of routine malaria control in Kita, Mali. Malar J. 2017;16:325.

13. Druetz T, Corneau-Tremblay N, Millogo T, Kouanda S, Ly A, Bicaba A, et al. Impact evaluation of seasonal malaria chemoprevention under routine program implementation: a quasi-experimental study in Burkina Faso. Am J Trop Med Hyg. 2017;98:524-33.

14. Ndiaye JLA, Ndiaye Y, Ba MS, Faye B, Ndiaye M, Seck A, et al. Seasonal malaria chemoprevention combined with community case management of malaria in children under 10 years of age, over 5 months, in south-east Senegal: a cluster-randomised trial. PLoS Med. 2016;16:e1002762.

15. Salissou I, Lamine MM, Ibrahim A, Djakou H, Maman LI. Estimation de l'impact en santé publique de la chimioprévention du paludisme saisonnier au Niger. Int J Biol Chem Sci. 2017;11:685-93.

16. Wilson AL. A systematic review and meta-analysis of the efficacy and safety of Intermittent preventive treatment of malaria in children (IPTC). PLOS ONE. 2011;6:e16976.

17. Cisse B, Ba EH, Sokhna C, Ndiaye JL, Gomis JF, Dial Y, et al. Effectiveness of seasonal malaria chemoprevention in children under ten years of age in Senegal: a stepped-wedge cluster-randomised trial. PLoS Med. 2016;13:e1002175.

18. Zongo I. Efficacy, safety, tolerability of dihydroartemisinine-piperaquine and sulfadoxine-pyrimethamine plus amodiaquine for seasonal malaria chemoprevention (SMC) in children in Burkina Faso. PhD thesis. London School of Hygiene \& Tropical Medicine; 2014.

19. Cairns M, Roca-Feltrer A, Garske T, Wilson AL, Diallo D, Milligan PJ, et al. Estimating the potential public health impact of seasonal malaria chemoprevention in African children. Nat Commun. 2012;3:881.

20. Meremikwu MM, Donegan S, Sinclair D, Esu E, Oringanje C. Intermittent preventive treatment for malaria in children living in areas with seasonal transmission. Cochrane Database Syst Rev. 2012;2:CD003756.

21. Tagbor H, Antwi GD, Acheampong PR, Bart Plange C, Chandramohan D, Cairns M. Seasonal malaria chemoprevention in an area of extended seasonal transmission in Ashanti, Ghana: an individually randomised clinical trial. Trop Med Int Health. 2016;21:224-35.

\section{Publisher's Note}

Springer Nature remains neutral with regard to jurisdictional claims in published maps and institutional affiliations. 